http://dx.doi.org/10.5012/bkcs.2014.35.9.2828

\title{
Fe(III)/Polydopamine-Mediated Capture and Release of Catecholic Compounds
}

\author{
Suyeob Kim, Taewoo Gim, Ji Woo Park, Jongnam Kim, and Sung Min Kang* \\ Department of Marine Bio-Materials \& Aquaculture, Pukyong National University, Busan 608-737, Korea \\ ${ }^{*}$ E-mail:smk12@pknu.ac.kr \\ ${ }^{\dagger}$ Department of IT Convergence and Application Engineering, Pukyong National University, Busan 608-737, Korea \\ Received March 17, 2014, Accepted May 12, 2014
}

Key Words : Polydopamine, Catechol, Capture

The special properties of some marine organisms such as underwater adhesive property of mussel $^{1}$ and silica-forming ability of diatom ${ }^{2}$ are related to proteins comprising nonnatural amino acids, and hence, various efforts have been made to isolate and identify these proteins. ${ }^{1-4}$ Boronate affinity chromatography (BAC) is a representative method to isolate proteins containing unnatural amino acid, 3,4dihydroxyphenyl-L-alanine (DOPA). ${ }^{3,4}$ In BAC, catecholcontaining molecules are selectively bound to the resin modified with phenylboronic acids, resulting in the purification of the molecules. ${ }^{5}$ Because of the presence of the catechol unit in DOPA, BAC has been frequently employed to purify DOPA-containing proteins. For example, BAC was used in the analysis of squid beak which is known as one of the hardest organic materials. ${ }^{3}$ Ali et al. performed various chemical analyses including BAC, and found that the squid beak consists of chitin, water, and DOPA-containing proteins. Although the exact role of DOPA-containing proteins has not been elucidated, the identification of the specific protein provided a meaningful insight to understand the extraordinary mechanical properties of squid beak. BAC has been also utilized in the analysis of sandcastle worm's underwater adhesion. ${ }^{4}$ Sandcastle worm is well-known that it utilizes the protein-based, underwater adhesive for the construction of its home. Zhao et al. identified the cysteinyldopa residues from the adhesive and proposed that the ad-

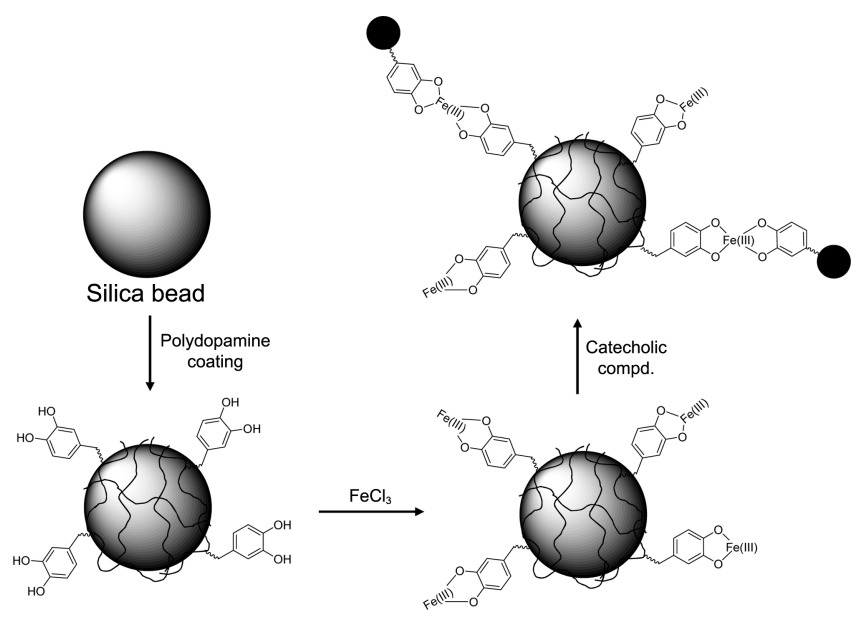

Figure 1. Schematic description of the Fe(III)/polydopaminemediated capture of catecholic compounds. hesive is hardened by crosslinking reaction between DOPA and cysteine groups in proteins. ${ }^{4}$ In the study, BAC also played a crucial role for identifying cysteinyl-dopa residues.

Albeit the successful use of BAC, it has a limitation that can only be performed under aqueous conditions, and therefore, development of a new method for the separation of catechol-containing molecules is required. Herein, we developed a mussel-inspired approach that captures catechol-containing molecules under non-aqueous conditions.

Mussel byssus cuticle which protects the inner core of the mussel byssus has gained a great deal of attention, because of its high extensibility combined with high stiffness and hardness. ${ }^{6-8}$ Harrington et al. reported that the aforementioned properties of the mussel byssus cuticle are attributable to complexation between catechol-containing proteins and $\mathrm{Fe}(\mathrm{III}) .{ }^{9}$ More specifically, $\mathrm{Fe}(\mathrm{III})$ can coordinate to three catechol moieties, forming a catechol-Fe(III)-catechol bridge, which is robust and reversible. ${ }^{10,11}$ Subsequently, this chelating property has been used for developing advanced techniques such as the preparation of self-healing gels ${ }^{12}$ and reversible layer-by-layer depositions. ${ }^{13}$ Given its wide applicability in various areas, we envisioned that the catechol-Fe(III) chelation can be utilized for the capture and release of catecholic compounds. Moreover, unlike BAC, catechol-Fe(III) chelation can be effected under non-aqueous conditions. ${ }^{13}$ Therefore, it is applicable to water-insoluble catechols.

Columns for catechol capture were prepared by using polydopamine (pDA)-coated silica beads. pDA coating, which is a well-known method to introduce catechols on solid substrates, ${ }^{14-17}$ was employed for the fabrication of catechol-immobilized silica beads. Subsequently, complexation with $\mathrm{Fe}(\mathrm{III})$ was carried out by treatment with an ethanolic solution of $\mathrm{FeCl}_{3}$. Finally, a second catechol molecule was captured by the column via the formation of a catechol-Fe(III)-catechol coordination bridge (Fig. 1). Each step was analyzed by X-ray photoelectron spectroscopy (XPS) using a flat Si substrate instead of silica beads (Fig. 2). pDA coating and Fe(III) complexation were confirmed from the new peaks at $400 \mathrm{eV}$ (Fig. 2(b)) and 710-730 eV (Fig. 2(c)), corresponding to N 1 s of pDA and Fe 2p, respectively. Quantitative analysis of surface chemical compositions was further investigated. After the pDA coating, the amounts of $\mathrm{C} 1 \mathrm{~s}$ and $\mathrm{N} 1 \mathrm{~s}$ increased from 9.06 to $60.75 \%$ and 0 to $5.87 \%$, respectively, with a concurrent decrease in 

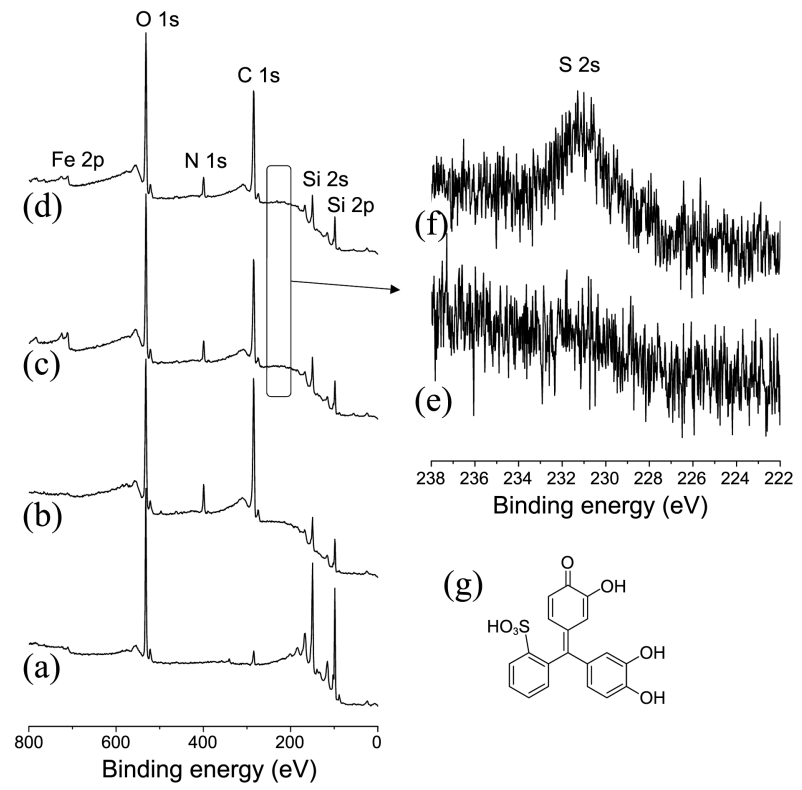

(g)

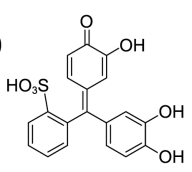

Figure 2. X-ray photoelectron spectra of (a) unmodified, (b) pDAcoated, (c) Fe(III)/pDA-coated, and (d) PCV/Fe(III)/pDA-coated Si substrates. High-resolution X-ray photoelectron spectra of (e) $\mathrm{Fe}(\mathrm{III}) / \mathrm{pDA}$-coated and (f) PCV/Fe(III)/pDA-coated Si substrates. (g) Chemical structure of PCV.

the amounts of Si $2 \mathrm{p}$ and O 1s from 61.11 to 11.29 and 29.82 to $21.6 \%$. In addition, the amount of $\mathrm{Fe} 2 \mathrm{p}$ increased from 0 to $1.33 \%$, after the $\mathrm{Fe}(\mathrm{III})$ complexation. All these observations indicate the successful pDA coating and $\mathrm{Fe}(\mathrm{III})$ complexation on the surface.

Pyrocatechol violet (PCV) was chosen as a model analyte for capture, because of its strong chromophoric ability (Fig. 2(g)). PCV strongly absorbs light in the visible region (440$450 \mathrm{~nm}$ ), and this optical property is advantageous for quantifying the amount of PCV captured by columns. The PCV capture was first analyzed by XPS. In the survey spectrum (Fig. 2(d)), characteristic peaks for PCV were not observed, due to the low content of PCV on surface: up to one monolayer of PCV can be captured by the surface, because of the limited reaction site. For characterizing PCV, XPS narrow scan of the surface was carried out before and after PCV capture (Figs. 2(e) and (f)). PCV-captured surface showed a new peak at $231 \mathrm{eV}$, corresponding to $\mathrm{S} 2 \mathrm{~s}$ of PCV, thereby confirming that PCV was successfully captured on the surface.

The capturing ability of the column was determined by UV-Vis spectroscopy (Fig. S1). $8 \mu \mathrm{mol}$ of PCV was loaded onto the column, and the amount of PCV eluted from the column (non-captured PCV) was quantified. As a control, same experiment was performed without Fe(III) complexation. The amount of non-captured PCV was calculated from standard curves constructed using PCV concentrations of 10 , 20,50 , and $70 \mu \mathrm{M}$. The capturing ability of the Fe(III)treated column and control was 7.26 \pm 0.31 and $2.26 \pm 1.17$ $\mu \mathrm{mol}$, respectively (Fig. 3). The capturing ability of the control might be due to $\pi-\pi$ interactions and hydrogen bonding between the catechol moieties. As expected, the Fe(III)

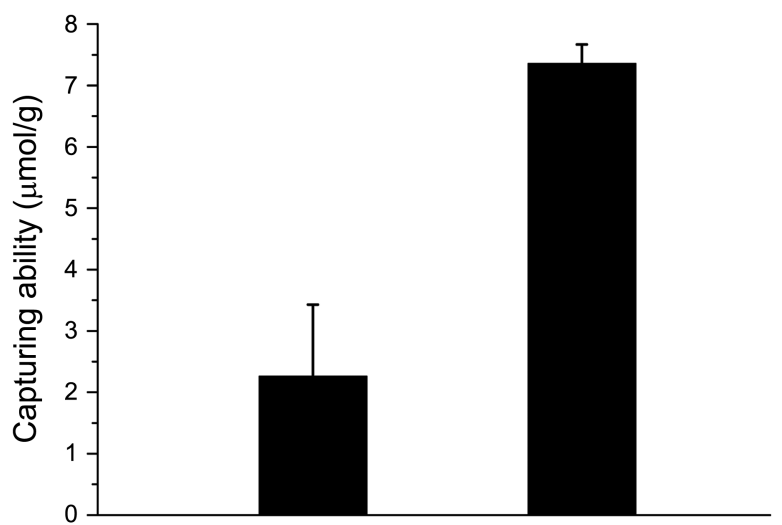

(a)

(b)

Figure 3. Capturing ability of columns packed with (a) pDAcoated silica beads (control) and (b) Fe(III)/pDA-coated silica beads.

complexation resulted in significant enhancement of the capturing ability of the column.

The release of captured PCV was demonstrated by ethylenediaminetetraacetic acid (EDTA) treatment. It is known that catechol-Fe(III) coordinate bond can be cleaved by the addition of a chelating agent. ${ }^{11,13}$ In this study, we utilized EDTA, the most common chelating agent for transition metals, and the removal of Fe(III) from the surface was verified by XPS. Figure S2 shows XPS survey scan of the EDTA-treated surface. The peak for Fe $2 p$ disappeared, indicating that $\mathrm{Fe}(\mathrm{III})$ was removed through chelation with EDTA. The PCV release experiment was then conducted. Captured PCV was allowed to separate from the column by EDTA treatment, and the released PCV was monitored by UV-Vis spectroscopy. Figure 4 shows an elution profile of PCV separated from the column. The release of PCV started upon EDTA treatment, and was completed after $60 \mathrm{~mL}$ elution, showing the good performance of the column in both capture and release of catecholic compounds.

In summary, a new method for the capture of catecholic compounds has been developed by inspiration from mussel byssus cuticle. Columns prepared using silica beads pre-

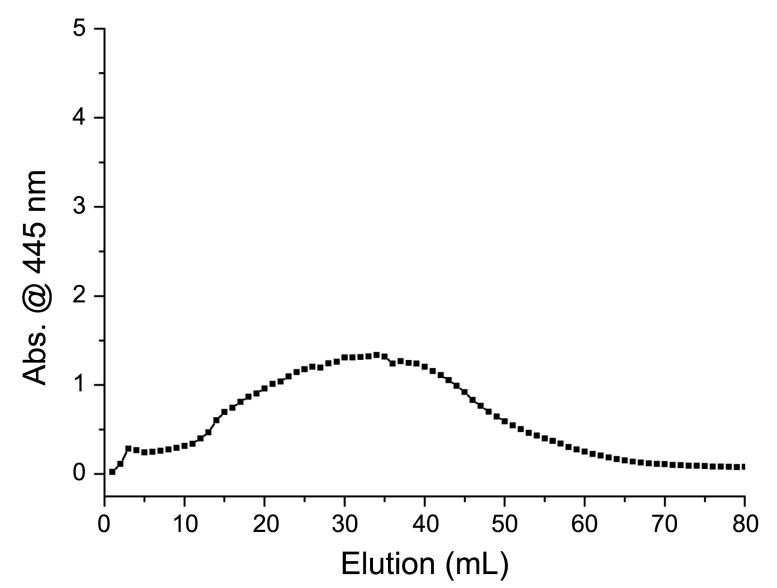

Figure 4. Elution profile of PCV separated from the column by EDTA treatment. 
treated with pDA and Fe(III) could capture catecholic analytes. Moreover, release of the captured compounds was demonstrated by the addition of a chelating agent, EDTA. The capturing ability mainly originated from the catechol-Fe(III) coordinate bond, which is cleavable by EDTA treatment. We believe that this technique for catechol capture and release can be used to purify and isolate catecholic compounds having antioxidizing and bioactive properties.

\section{Experimental}

Materials. Dopamine hydrochloride (98\%, Aldrich), trizma base (99\%, Sigma), trizma $\mathrm{HCl}$ (99\%, Sigma), iron(III) chloride hexahydrate $\left(\mathrm{FeCl}_{3} \cdot 6 \mathrm{H}_{2} \mathrm{O}, 97 \%\right.$, Sigma-Aldrich), pyrocatechol violet (99\%, Sigma-Aldrich), silica gel (99\%, Sigma-Aldrich), and absolute ethanol (Merck) were used as received.

pDA Coating on Silica Beads. pDA coating was performed by combining silica gel (200-425 mesh particle size, 10 $\mathrm{mg} / \mathrm{mL})$ with a dopamine solution $(10 \mathrm{mM}, \mathrm{pH} 8.5)$ at room temperature. After 1-hr coating, pDA-coated silica beads were separated from the suspension and washed several times by centrifuging/redispersing in water and ethanol.

Column Preparation and Fe(III) Complexation. $1 \mathrm{~g}$ of pDA-coated silica beads were suspended in ethanol and poured into the column. After packing, an ethanolic solution of $\mathrm{FeCl}_{3}(10 \mathrm{mM}, 10 \mathrm{~mL})$ was added to the column for the complexation reaction. Residual $\mathrm{FeCl}_{3}$ was then washed away with ethanol ( $50 \mathrm{~mL})$.

Capture and Release of Pyrocatechol Violet (PCV). 8 $\mu \mathrm{mol}$ of PCV was dissolved in ethanol and passed through the column. Non-captured PCV was monitored by UV-Vis spectroscopy. Captured molecules were then released by eluting with an EDTA solution ( $1 \mathrm{mM}, \mathrm{pH} 3.5)$. Typically, the eluent was added onto a column and allowed to flow forced by gravity. The elution of the PCV was analyzed by UV-Vis spectroscopy, and the detection wavelength was 445 $\mathrm{nm}$.
Characterizations. X-ray photoelectron spectra were obtained using a MultiLab 2000 system (Thermo VG Scientific, UK) with a Mg K $\alpha$ X-ray source and ultrahigh vacuum $\left(\sim 10^{-10}\right.$ mbar). UV-Vis spectroscopy was performed on a LAMBDA $35 \mathrm{UV} /$ Vis spectrophotometer (Perkin Elmer).

Acknowledgments. This work was supported by a Research Grant of Pukyong National University (2014 year).

Supporting Information. Figure S1 and 2.

\section{References}

1. Waite, J. H.; Qin, X. Biochemistry 2001, 40, 2887.

2. Kröger, N.; Deutzmann, R.; Sumper, M. Science 1999, 286, 1129.

3. Miserez, A.; Schneberk, T.; Sun, C.; Zok, F. W.; Waite, J. H. Science 2008, 319, 1816.

4. Zhao, H.; Sun, C.; Stewart, R. J.; Waite, J. H. J. Biol. Chem. 2005, $280,42938$.

5. Li, H.; Liu, Z. Trends Anal. Chem. 2012, 37, 148.

6. Holten-Andersen, N.; Fantner, G. E.; Hohlbauch, S.; Waite, J. H.; Zok, F. W. Nature Mater. 2007, 6, 669.

7. Holten-Andersen, N.; Mates, T. E.; Toprak, M. S.; Stucky, G. D.; Zok, F. W.; Waite, J. H. Langmuir 2008, 25, 3323.

8. Holten-Andersen, N.; Zhao, H.; Waite, J. H. Biochemistry 2009, 48, 2752.

9. Harrington, M. J.; Masic, A.; Holten-Andersen, N.; Waite, J. H.; Fratzl, P. Science 2010, 328, 216.

10. Wilker, J. J. Angew. Chem. Int. Ed. 2010, 49, 8076.

11. Zeng, H.; Hwang, D. S.; Israelachvili, J. N.; Waite, J. H. Proc. Natl. Aca. Sci. USA 2010, 107, 12850.

12. Holten-Andersen, N.; Harrington, M. J.; Birkedal, H.; Lee, B. P.; Messersmith, P. B.; Lee, K. Y. C.; Waite, J. H. Proc. Natl. Aca. Sci. USA 2011, 108, 2651.

13. Kim, S.; Kim, D. S.; Kang, S. M. Chem. Asian J. 2014, 9, 63.

14. Lee, H.; Dellatore, S. M.; Miller, W. M.; Messersmith, P. B. Science 2007, 318, 426.

15. Kang, S. M.; You, I.; Cho, W. K.; Shon, H. K.; Lee, T. G.; Choi, I. S.; Karp, J. M.; Lee, H. Angew. Chem. Int. Ed. 2010, 49, 9401.

16. Ryu, J.; Ku, S. H.; Lee, H.; Park, C. B. Adv. Func. Mater. 2010 , 20, 2132.

17. Hong, S.; Na, Y. S.; Choi, S.; Song, I. T.; Kim, W. Y.; Lee, H. $A d v$. Func. Mater. 2012, 22, 4711. 